\title{
Physical model of Seebeck coefficient under surface dipole effect in organic thin-film transistors
}

\author{
Nianduan $\mathrm{Lu}^{1)}$, Ling $\mathrm{Li}^{2)}$, Writam Banerjee, and Ming Liu
}

Key Laboratory of Microelectronics Devices and Integrated Technology, Institute of

Microelectronics, Chinese Academy of Sciences, No.3, Bei-Tu-Cheng West Road,

Beijing 100029, China

\begin{abstract}
The surface dipole effect can play an important role in the performance of charge carrier transport in organic thin-film transistors (OTFTs). In this work, we propose a physical model of Seebeck coefficient based on variable-range hopping theory in OTFTs to characterize carrier thermoelectric transport. The model effectively explains the influence of a dipole on the carrier density, energetic disorder and temperature dependence of the Seebeck effect. The gate-voltage and temperature dependence of the Seebeck effect are remarkably enhanced by a dipole, while the energetic disorder exhibits a weak dependent nature. The Seebeck coefficients calculated in this study and those obtained experimentally in a previous study were found to be in good agreement.
\end{abstract}

\footnotetext{
${ }^{1)}$ Electronic mail address: lunianduan@ime.ac.cn

${ }^{2)}$ Electronic mail address: lingli@ime.ac.cn
} 


\section{INTRODUCTION}

Organic thin-film transistors (OTFTs) are of growing interest due to their potential applications in areas such as electronics, optoelectronics and displays [1,2]. Following years of research efforts, OTFTs have been developed from a laboratory curiosity into materials suitable for advanced applications in commercially viable technology, such as flexible displays, sensors and radiofrequency identification (RFID) tags [3-5]. OTFTs usually operate in accumulation mode. Because the charge carrier transport is typically confined to the first few monolayers of the organic semiconductors adjacent to the gate dielectric, the transistor performance is greatly influenced by the interface [6,7]. An OTFT generally includes two critical interfaces: the interface between the gate dielectric and the organic semiconductor, and the interface between the source/drain contacts and the organic semiconductor. In physics, an electric dipole refers to a separation of electric charge. The electric dipole moment is a measure of the separation of positive and negative electrical charges in a system of electric charges. A surface dipole is a neutral charge with an electric dipole moment per unit area directed perpendicular to the surface. It has been demonstrated that the interfaces between organic semiconductors and metals are generally characterized by the presence of a relatively dipole which is confined to a thin interfacial layer [8]. Otherwise, the surface dipole effect, which is brought about by introducing self-assembled monolayers (SAMs) with different functional groups, can efficiently improve the electrical performance of OTFTs $[9,10]$. Moreover, dipoles at the insulator/semiconductor interface in OTFTs have been studied to achieve better 
control of the channel conductance and carrier density $[9,11]$.

In general, temperature and gate-voltage dependent field-effect mobility is used to characterize the charge transport of OTFTs $[12,13]$. On the other hand, the charge carrier transport in OTFTs can also be described by a thermally activated hopping process between localized states or band-like transport [14-17]. However, when the channel length is scaled down and the transistor operates at low fields, interface contact resistance dramatically affects the charge transport, and therefore the performance of OTFTs [18]. To eliminate the effect of contact resistance on the device performance, new characterization methods such as the Seebeck effect have been developed to investigate the charge carrier transport [19]. In general, the electric field induced by the Seebeck effect is parallel to the applied temperature [20]. The Seebeck effect is described by the Seebeck coefficient (S), which is given by the relation $S=-V_{\text {therm }} / \Delta T$, where the thermoelectric voltage $V_{\text {therm }}$ arises when the two ends of a sample are held at different temperatures $(\Delta T)[21]$. The Seebeck effect is very effective at uncovering the intrinsic charge carrier transport process, as the Seebeck voltage is independent of the interfacial contact. The Seebeck effect of organic transistors has been widely investigated both theoretically and experimentally, and the carrier thermoelectric transport has been received much attention $[14,21,22]$. However, in OTFTs, the effect of dipoles on the Seebeck effect is still ambiguous and requires more in-depth investigation. Because the carrier transport characteristics in organic semiconductors can be remarkably affected by a dipole [23-25], it is imperative to understand their effects on thermoelectric transport for the better design 
of high-performance OTFTs.

In this work, we propose a Seebeck effect model based on variable-range hopping theory to analyze carrier thermoelectric transport in OTFTs. The effect of gate-voltage, dipole, energetic disorder and temperature on the Seebeck effect will be discussed in details based on the proposed physical model.

\section{THEORETICAL MODEL}

It is widely accepted that charge carrier transport in disordered organic semiconductors is governed by variable-range hopping (VRH) among the system of localized states. The Gaussian density of states (DOS) is usually assumed to describe the energy distribution of localized states as follows:

$$
g(E)=\frac{N_{t}}{\sqrt{2 \pi} \sigma_{0}^{2}} \exp \left(-\frac{E^{2}}{2 \sigma_{0}^{2}}\right)
$$

where $N_{t}$ is the number of states per unit volume, $E$ is the energy and $\sigma_{0}$ indicates the width of the DOS.

A dipole moment $P$ for a charge pair is $\vec{P}=E \vec{L}$, where the vector $\vec{L}$ points from the negative charge to the positive charge. As a given dipole moment affects the system, the probability density $w(r)$ is determined by the Poisson distribution as [24]

$$
w(r)=4 \pi r^{2} N_{t} \exp \left(-\frac{4}{3} \pi r^{3} N_{t}\right)
$$

where $r$ is the distance between the carrier and center of a dipole.

For a carrier trapped by a localized state, the potential energy of the interaction between the carrier and dipole moment can be written as [26]

$$
E_{c c}=-\frac{q P \cos \theta}{4 \pi \varepsilon_{s} r^{2}}
$$


where $q$ is the elementary charge, $\varepsilon_{S}$ is the dielectric constant, and $\theta$ is the angle between vectors $\vec{L}$ and $\vec{r}$. The previous work indicated that surface dipole effect could induce a deviation in the DOS distribution and broaden the tail states of the DOS in organic semiconductors [24,25]. Figure 1 displays the device structure of OTFTs and schematic diagrams of variable-range hopping theory for the cases of a simple Gaussian DOS and a deviated DOS induced by surface dipole effect.

Because the dipole orientation is random, the total energy of the interaction between the charge and total dipole moment of the interface is calculated as

$$
E_{c}=\int_{-\pi / 2}^{\pi / 2} \int_{0}^{\pi / 2} E_{c c} d \theta d \varphi
$$

For a localized state, the total energy $E$ of the hopping site can be obtained as $E=E_{c}+E_{0}$, where $E_{0}$ is the intrinsic disorder energy. Finally, the deviated DOS with the dipole effect can be rewritten as [24]

$$
g_{d}(E)=2 \pi N_{t}\left(\frac{q P}{8 \pi \varepsilon_{s}}\right)^{\frac{3}{2}} \int_{-\infty}^{0} \exp \left(-\frac{4 \pi N_{t}}{3}\left(-\frac{q P}{8 \pi \varepsilon_{s} E_{c}}\right)^{\frac{3}{2}}\right)\left(-E_{c}\right)^{-\frac{5}{2}} g\left(E-E_{c}\right) d E_{c}
$$

Then, the carrier concentration is written as

$$
n=\int_{-\infty}^{\infty} g_{d}(E) f(E, \varphi(x)) d E,
$$

here $f(E, \varphi(x)$ is the Fermi-Dirac distribution, $\varphi(x)$ is the gate-induced potential.

In a transistor, carriers accumulate in the semiconductor-insulator interface under the effect of gate voltage, and the density of an accumulated charge carrier decreases with distance $x$ [16]. The gate-induced potential $\varphi(x)$ shifts the difference between the transport band edge and the Fermi level. Therefore, the quasi-Fermi level $E_{f}(x)$ is

$$
E_{f}(x)=E_{f 0}+q \varphi(x)
$$


The variation of $\varphi(x)$ with respect to the distance $x$ is determined by the Poisson equation as [13]

$$
F(x)^{2}=\frac{2 q}{\varepsilon_{s}} n=\frac{2 q}{\varepsilon_{s}} \int_{0}^{\varphi(x)} \int_{-\infty}^{\infty} g_{d}(E) f(E, \varphi(x)) d \varphi d E,
$$

where $\varepsilon_{s}$ is the dielectric constant of the organic semiconductor and $F(x)$ is the electric field perpendicular to the interface. At the semiconductor-insulator interface, the electric field $F(0)$ can be calculated by the gate voltage $V_{g}$ and insulator capacitance per unit area $C_{i}$ through Gaussian law as

$$
V_{g}-V_{f b}-\varphi_{s}=\frac{1}{C_{i}} \sqrt{2 q \varepsilon_{s} \int_{0}^{\varphi_{s}} \int_{-\infty}^{\infty} \frac{g(E)}{1+\exp \left(E-E_{f}\right) / k_{B} T} d \varphi d E}
$$

where $V_{g}$ is the gate voltage, $V_{f b}$ is the flat-band voltage, $C_{i}$ is the insulator capacitance per unit area, $\varphi_{s}$ is the potential at the semiconductor-insulator interface.

Based on the generalized Einstein relation [28], the energy-dependent carrier mobility is written as follows

$$
\mu(E)=\eta \frac{q R(E)^{2} v_{e s c}(E)}{k_{B} T\left[1+\exp \left(\frac{E_{f}-E}{k_{B} T}\right)\right]} .
$$

Monroe had reported that the carrier transport includes two thermalization mechanisms: (a) hopping directly to deeper states; (b) thermal excitation to shallower states (transport states), transport, and subsequent retrapping at deeper states [29]. Therefore, the escape rate for carriers will cover these two transport processes, i.e., the carrier transition rate from a site with energy $E$ to another site with a different energy is given by the sum of average downward and upward hopping rates [30]. Based on Ambegaokar et al. [31], the intrinsic transition rate for a carrier hopping from an initial site $i$ to an empty site $j$ is expressed by $\gamma_{i j}=\gamma\left(R_{i j}, E_{i}-E_{j}\right)$. The average transition rate from site $i$ to site $j$ is then 


$$
v_{i j}=<n_{i}\left(1-n_{j}\right) \gamma_{i j}>
$$

where $n_{i}$ and $n_{j}$ are the occupation numbers, respectively. The energy dependence of $\gamma_{i j}$ is then a good approximation to write as

$$
\gamma_{i j}= \begin{cases}\gamma_{0} \exp \left(-2 \alpha R_{i j}-\frac{E_{j}-E_{i}}{k_{B} T}\right), & \text { for } E_{j}>E_{i} \\ \gamma_{0} \exp \left(-2 \alpha R_{i j}\right) . & \text { for } E_{j}<E_{i}\end{cases}
$$

With $\gamma_{0}$ is some constant which depends on the electron-phonon coupling strength, $\alpha$ is the inverse localized length, $R_{\mathrm{ij}}$ is the hopping distance, $E_{\mathrm{i}}$ and $E_{\mathrm{j}}$ are the energies at sites $i$ and $j$, respectively, and $k_{B}$ is the Boltzmann constant. Therefore, based on the energy dependence of $\gamma_{i j}$, the total escape rate is expressed as

$$
v_{e s c}=v_{0} \exp \left(-r_{i j}\right)=v_{0} \begin{cases}\exp \left(-2 \alpha R_{i j}-\frac{E_{j}-E_{i}}{k_{B} T}\right), & E_{j}-E_{i}>0 \\ \exp \left(-2 \alpha R_{i j}\right) . & E_{j}-E_{i}<0\end{cases}
$$

where $v_{0}$ is the attempt-to-jump frequency.

The average hopping range of the carriers at energy $E$ is given by [32]

$$
R(E)=\left[\frac{4 \pi}{3 B_{c}} \int_{-\infty}^{E} g_{d}(E)(1-f(E)) d E\right]^{-\frac{1}{3}} .
$$

Here, the numerical value of parameter $B_{c}$ is determined to be 2.7 according to percolation criteria [32,33], $f(E)=1 /\left(1+\exp \left(E-E_{f}(x)\right)\right.$ is the Fermi-Dirac distribution, $1-f(E)$ is the probability that the final site is empty.

Finally, substituting Eqs. (6), (7), (13) and (14) into Eq. (10), the energy-dependent carrier mobility under the gate-induced potential $\varphi(x)$ is calculated as

$$
\mu(E, \varphi(x))=\frac{\eta q v_{0}}{k_{B} T}[1-f(E, \varphi(x))] R(E)^{2} \exp \left(-r_{i j}\right),
$$

According to the literature [34], a general expression of the Seebeck coefficient $S$ can be written as 


$$
S=\frac{1}{q T} \frac{\int\left(E-E_{f}\right) \sigma(E) d E}{\int \sigma(E) d E} .
$$

From Eq. (16), one can easily calculate the Seebeck coefficient if the energy-dependent conductivity $\sigma(E)$ can be calculated.

Based on a Kubo-Greenwood type calculation of conductivity, the total conductivity in the gate-induced potential $\varphi(x)$ can be written as [35]

$$
\sigma=\int \sigma(E) d E=\int_{0}^{\varphi_{s}} \int_{-\infty}^{0} q g_{d}(E) \mu(E, \varphi(x)) f(E, \varphi(x)) d E d \varphi(x),
$$

where the surface potential $\varphi_{s}$ can be solved according to the method previously described in the literature [13]. Then, the energy-dependent conductivity can be calculated as

$$
\sigma(E)=\int_{0}^{\varphi_{s}} q g_{d}(E) \mu(E, \varphi(x)) f(E, \varphi(x)) d \varphi(x)
$$

Finally, the Seebeck coefficient can be expressed as

$$
\left\{\begin{array}{l}
\mathrm{S}=\frac{1}{q T} \frac{\int_{0}^{\varphi_{S}} \int_{-\infty}^{0}\left(E-E_{f 0}-q \varphi(x)\right) g_{d}(E) A(x) B(E) d E d \varphi(x)}{\int_{0}^{\varphi_{S}} \int_{-\infty}^{0} g_{d}(E) A(x) B(E) d E d \varphi(x)} \\
A(x)=f(E, \varphi(x))^{2} \exp \left(E-E_{f 0}-q \varphi(x)\right) \\
B(E)=R(E)^{2} \exp \left(-r_{i j}\right)
\end{array}\right.
$$

\section{RESULTS AND DISCUSSION}

In order to prove the validity of the model, we first compare the theoretical calculations with experimental data for PEDOT:Tos [36], and for a high-quality rubrene single crystal [14], respectively, as shown in Fig. 2. To convert oxidation level to carrier density for PEDOT:Tos, we used the same approach with the literature [30], and the carrier density here is calculated by using Eq. (6). The fitting parameters are similar to the Ref., such as the total DOS $N_{t}=1 \times 10^{21} \mathrm{~cm}^{-3}[30]$ and $N_{t}=1.4 \times 10^{21}$ $\mathrm{cm}^{-3}[14]$. The good agreement between the calculation result and the experimental 
data is observed.

To demonstrate the effect of a dipole on the Seebeck effect in OTFTs, then we will discuss the influence of a dipole moment on the gate-voltage, energetic disorder and temperature dependence of the Seebeck coefficient. Figure 3 shows the gate-voltage dependence of the Seebeck coefficient at different dipole moments. The parameters other than $T$ are obtained using a transport model previously described in the literature $[13,37,38]$. Figure 3 clearly shows that the Seebeck coefficient depends strongly on the gate-voltage under the surface dipole effect. More remarkably, the dependence of the Seebeck coefficient on the gate-voltage becomes enhanced when the dipole moment increases. In general, the deep energy states become gradually occupied as the gate-voltage is increased (a larger gate-voltage corresponds to a high density). Because of the deep level trapping of charge carriers, the occupied probability of charge carriers in shallow energy states is decreased as the gate-voltage is increased. It is well known that the Seebeck coefficient is proportional to the flow of entropy transported by an electrical current. With an increase in the gate voltage, the carrier concentration in the system increases, resulting in a decrease in the flow of entropy transported, which in turn decrease the Seebeck coefficient. More importantly, when a dipole moment increases, the DOS can be broadened, as well as the increase of disorder [24]. The broadened DOS and increased disorder can shift the Fermi level upward [39], which will result in the decrease of the occupied probability of charge carriers in shallow energy states. According to the results from W. Chr. Germs et al. [40], within the variable-range hopping (VRH) transport, the Seebeck coefficient is 
proportional to the Fermi level $\left(E_{f}\right)$, such as $S \propto \frac{E_{t}-E_{f}}{q T}$ (here $E_{t}$ is the transport energy). Otherwise, although the carrier concentration increases with the increase of gate voltage, the increasing carriers will prior to be trapped by deep energy states when Fermi level shift upward. Therefore the increasing carriers don't contribute to the flow of entropy transported by an electrical current. Consequently, the gate-voltage dependence of the Seebeck coefficient becomes much more intense when the dipole moment is increased.

Figure 4 shows the dependence of the Seebeck coefficient on the dipole moment for different energetic disorders. The results in Fig. 4 demonstrate that the Seebeck coefficient varies non-monotonically as the dipole moment is increased. For example, as $\mathrm{P}<5 \mathrm{D}$, the Seebeck coefficient decreases with the increase of the dipole moment, while increases for P > 5 D. As mentioned above, the dipole moment can broaden DOS and increase the disorder, which results in an increasingly large number of shallow sites into deep states and accordingly decrease the mobility. Therefore the Seebeck coefficient decreases remarkably with the increase of the dipole moment in the lower dipole moment. When the dipole moment is larger, such as P > $5 \mathrm{D}$, the strength of Coulomb interaction between carrier and dipole will increase and hence promote the carrier transport. As a result, the Seebeck coefficient will increase with the increase of the dipole moment, as the surface dipole effect is larger, which is consistent with the results reported at which increasing the strength of the Coulomb interaction can increase the Seebeck coefficient [41].

Figure 5 shows the dependence of the Seebeck coefficient on energetic disorder 
for different dipole moments. One can see that the Seebeck coefficient exhibits a strong dependence on energetic disorder; specifically, the Seebeck coefficient increases with increasing energetic disorder, which is consistent with the results of G. Kim et al. for $\mathrm{P}=0 \mathrm{D}$ [30]. Moreover, the dependence of Seebeck coefficient on energetic disorder becomes weaker as the dipole moment is increased. These results suggest that the dipole effect can distinctly decrease the dependence of the Seebeck coefficient on energetic disorder.

Finally, we will discuss the temperature dependence of the Seebeck coefficient and compare our theoretical calculations with experimental data (from Ref. 21). The results are displayed in Fig. 6, which was generated using fitting parameters of $N_{t}=1.75 \times 10^{19} \mathrm{~cm}^{-3}, \alpha^{-1}=1.5 \mathrm{~nm}, C_{i}=1 \times 10^{-4} \mathrm{~F} / \mathrm{m}^{2}, \sigma_{0} / \mathrm{k}_{\mathrm{B}} \mathrm{T}=4, \varepsilon_{s}=3, E_{f 0}=-0.52 \mathrm{eV}$, and $V_{g}-V_{f b}=6 \mathrm{~V}$. The parameters $N_{t}, \alpha^{-1}, C_{i}, \sigma_{0} / \mathrm{k}_{\mathrm{B}} \mathrm{T}, \varepsilon_{s}=3$ and $E_{f 0}=-0.52 \mathrm{eV}$ are similar to those used in transport models described in the literature $[13,37,38]$. The samples were fabricated by standard high-vacuum deposition techniques. To explore possible interfacial effects (such as the surface dipole effect) associated with the surface treatment of the oxide layer under various plasma exposure conditions, a SAM was deposited prior to pentacene growth. Plasma treatment with argon was performed at a radio frequency of $10 \mathrm{~W}$ for $5 \mathrm{~min}$ at a constant gas pressure of 0.1 mbar. The oxygen plasma treatment was performed under the same conditions. The top source and drain gold contacts were evaporated through a shadow mask at the temperature of liquid nitrogen. The channels were fabricated with a width of $6 \mathrm{~mm}$ and a length of 50 or $100 \mu \mathrm{m}$. The experimental data were measured by fixing the sample to two copper 
heating blocks in a top-contact thin (5-, 10-, and 100-nm thick active layers) pentacene transistor. The temperature was measured with a differential $\mathrm{NiCr} / \mathrm{CuNi}$ thermocouple adhered to the gold contacts with a thermally stable resin. One can see that the experimental results agree better with the simulation performed at $P=1 D$, compared with that performed at $\mathrm{P}=0 \mathrm{D}$. It is interesting to note that, the dependence of the Seebeck coefficient on the temperature is stronger when the dipole moment is larger, which shows that the surface dipole effect could change the thermally activated process during the charge carrier transport. However, the enhancement of the temperature dependence is attributed to the increase in energetic disorder, because the increase in the dipole moment can broaden the DOS and increase the energetic disorder $[24,42]$.

As mentioned above, the surface dipole effect can play an important role in the Seebeck effect of OTFTs. To better understand the effect of a dipole on the Seebeck coefficient from an experimental approach, one can measure the Seebeck coefficient in OTFTs with organosilane SAMs various materials, or with varying thickness because the interface dipole can be introduced by depositing the SAMs with different functional groups prior to pentacene growth $[9,21,23,43]$. In addition, because among many polymers investigated, the indacenodithiophene-co-benzothiadiazole (IDTBT) was found to have the lowest energetic disorder which approaching disorder-free transport [19], one can measure the Seebeck coefficient with the effect of isolate dipole by depositing the SAMs or not in IDTBT OTFTs. 


\section{CONCLUSION}

In summary, we have proposed a physical model based on variable-range hopping theory for expressing the Seebeck coefficient in OTFTs that takes into account the surface dipole effect. The dependence of the Seebeck coefficient on the gate-voltage, dipole effect, energetic disorder, and temperature has been discussed. The Seebeck coefficient varied non-monotonically as the dipole moment is increased. Additionally, the surface dipole effect can remarkably enhance the gate-voltage and temperature dependence, as well as weaken the energetic disorder dependence of the Seebeck coefficient. Finally, our theoretical calculation are in good agreement with the experimental results previously obtained on the temperature dependence of the Seebeck coefficient under the influence of the surface dipole effect in an organic thin-film transistor.

\section{ACKNOWLEDGES}

This work was supported in part by the Opening Project of Key Laboratory of Microelectronics Devices and Integrated Technology, Institute of Microelectronics, Chinese Academy of Sciences, in part by National Science Foundation of China (NSFC) under Grant No. 61574166 and 61306117, in part by Beijing Training Project for the Leading Talents in S\&T under Grant Z151100000315008, the National 973 Program under Grant 2013CBA01604 and Grant 2013CB933504, the Strategic Priority Research Program of the Chinese Academy of Sciences under Grant No. XDB12030400. 


\section{References:}

[1] Y. B. Yuan, G. Giri, A. L. Ayzner, A. P. Zoombelt, S. C. B. Mannsfeld, J. H. Chen, D. Nordlund, M. F. Toney, J. S. Huang, and Z. N. Bao, Nat. Comm., 5, 3005 (2004)

[2] M. Gsänger, E. Kirchner, M. Stolte, C. Burschka, V. Stepanenko, J. Pflaum, and F. Würthner, J. Am. Chem. Soc., 136, 2351 (2014).

[3] H. Klauk, Chem. Soc. Rev., 39, 2643 (2009).

[4] Y. Fujisaki, H. Koga, Y. Nakajima, M. Nakata, H. Tsuji, T. Yamamoto, T. Kurita, M. Nogi, and N. Shimidzu, Adv. Funct. Mater., 24, 1657 (2014).

[5] Z. Khozaee, L. S. Vargas, A. N Cammidge, M. J. Cook, and A. K. Ray, Mater. Res. Express, 2, 096305 (2015).

[6] M. Mottaghi, and G. Horowitz, Organic Electronics, 7, 528 (2006).

[7] A. Pivrikas, M. Ullah, Th. B. Singh, C. Simbrunner, G. Matt, H. Sitter, N. S. Sariciftci, Organic Electronics, 12, 161-168 (2011).

[8] R. Abdur, Y. K. Lee, K. Jeong, H. S. Nam, Y. H. Kim, J. Kim, J. Lee, Organic Electronics, 26, 8-14 (2015).

[9] S. Kobayashi, T. Nishikawa, T. Takenobu, S. Mori, T. Shimoda, T. Mitani, H. Shimotani, N. Yoshimoto, S. Ogawa, and Y. Iwasa, Nat. Mater., 3, 317 (2004).

[10] S. G. J. Mathijssen, E. C. P. Smits, P. A. van Hal1, H. J. Wondergem, S. A. Ponomarenko, A. Moser, R. Resel, P. A. Bobbert, M. Kemerink, R. A. J. Janssen, and D. M. D. Leeuw, Nat. Nanotech., 4, 674 (2009).

[11] K. P. Pernstich, S. Haas, D. Oberhoff, C. Goldmann, D. J. Gundlach, B. Batlogg, 
A. N. Rashid, and G. Schitter, J. Appl. Phys., 96, 6431 (2004).

[12] M. Ullah, Th. B. Singh, H. Sitter, and N. S. Sariciftci, Appl. Phys. A: Mater. Sci. Process., 97, 521 (2009).

[13] L. Li, K. S. Chung, and J. Jang, Appl. Phys. Lett., 98, 023305 (2011).

[14] K. P. Pernstich, B. Rössner, and B. Batlogg, Nat. Mater., 7, 321 (2008).

[15] V. I. Arkhipov, E. V. Emelianova, A. Kadashchuk, and H. Bässler, Chem. Phys., 266, 97-108 (2001).

[16] N. D. Lu, L. Li, P. X. Sun, W. Banerjee, and M. Liu, J. Appl. Phys., 116, 104502 (2014)

[17] J. Puigdollers, A. Marsal, S. Cheylan, C. Voz, R. Alcubilla, Organic Electronics, 11, 1333-1337 (2010).

[18] W. P. Wu, Y. Q. Liu, Y. Wang, H. X. Xi, X. K. Gao, C. G. Di, G. Yu, W. Xu, and D. B. Zhu, Adv. Funct. Mater., 18, 810 (2008).

[19] D. Venkateshvaran, M. Nikolka, A. Sadhanala, V. Lemaur, M. Zelazny, M. Kepa, M. Hurhangee, A. J. Kronemeijer, V. Pecunia, I. Nasrallah, I. Romanov, K. Broch, I. McCulloch, D. Emin, Y. Olivier, J. Cornil, D. Beljonen, and H. Sirringhaus, Nature, $\mathbf{5 1 5}, 384(2014)$

[20] D. Venkateshvaran, A. J. Kronemeijer, J. Moriarty, D. Emin, and H. Sirringhaus, APL Mater., 2, 032102 (2014).

[21] N. D. Lu, L. Li, and M., Liu, Organic Electronics, 16, 113-117 (2015).

[22] W. Chr. Germs, K. Guo, R. A. J. Janssen, and M. Kemerink, Phys. Rev. Lett., 109, $016601(2012)$ 
[23] Y. Jang, J. H. Cho, D. H. Kim, Y. D. Park, M. Hwang, and K. Cho, Appl. Phys. Lett., 90, 132104 (2007).

[24] L. Li, N. D. Lu, and M. Liu, Appl. Phys. Lett., 103, 253303 (2013).

[25] T. Richards, M Bird, and H. Sirringhaus, J. Chem. Phys., 128, 234905 (2008).

[26] C. Celle, C. Suspène, M. Ternisien, S. Lenfant, D. Guérin, K. Smaali, K. Lmimouni, J. P. Simonato, D. vuillaume, Organic Electronics, 15, 729-737 (2010).

[27] V. I. Arkhipov and P. Heremans, E. V. Emelianova, and H. Bässler, Phys. Rev. B, 71, 045214 (2005).

[28] R. Schmechel, J. Appl. Phys., 93, 4653 (2003).

[29] D. Monroe, Phys. Rev. Lett., 54(2), 146-149 (1985).

[30] G. Kim, and K. P. Pipe, Phys. Rev. B, 86, 085208 (2012).

[31] V. Ambegaokar, B. I. Halperin, and J. S. Langer, Phys. Rev. B, 4(8), 2612-2620 (1971).

[32] S. D. Baranovskii, Phys. Status Solidi B, 251, 487 (2014).

[33] I. I. Fishchuk, V. I. Arkhipov, A. Kadashchuk, P. Heremans, and H. Bässler, Phys. Rev. B, 76, 045210 (2007).

[34] H. Fritzsche, Solid State Commun., 9, 1813 (1971).

[35] P. Nagel, Electronic Transport in Disordered Semiconductors, Chapter 5 in Amorphous Semiconductors, topics in Applied Physics, Vol. 36, edited by M. H. Brodsky (Springer-Verlag, Berlin, 1979).

[36] O. Bubnova, Z. U. Khan, A. Malti, S. Braun, M. Fahlman, M. Berggren, and X. Crispin, Nat. Mater., 10, 429(2011). 
[37] https://www.repository.cam.ac.uk/handle/1810/245517.

[38] J. P. Gonzalez-Vazquez, J. A. Anta, and J. Bisquert, Phys. Chem. Chem. Phys., 11, 103259-10367 (2009).

[39] T. H. Nguyen, S. K. O’Leary, J. Appl. Phys., 88, 3479 (2000).

[40] W. Chr. Germs, K. Guo, R. A. J. Janssen, and M. Kemerink, Phys. Rev. Lett., 109, 016601 (2012).

[41] http://arxiv.org/ftp/arxiv/papers/1410/1410.4907.pdf.

[42] Ralph H. Young, and John A. Sinicropi, J. Phys. Chem., 99, 9497-9506 (1995).

[43] X. Crispin, V. Geskin, A. Crispin, J. Cornil, R. Lazzaoni, W. R. Salaneck, J. L. Bredas, J. Am. Chem. Sco., 124, 8131 (2002). 


\section{Figure Captions}

Figure 1. (a) The device structure of OTFT with the dipole, and (b) schematic diagrams of variable-range hopping theory with a simple Gaussian DOS (GDOS) or a deviated DOS (DDOS) induced by dipole, respectively.

Figure 2. Comparison between calculated and experimental values of the Seebeck coefficient for PEDOT:Tos and Rubrene, respectively. The input parameters for PEDOT:Tos are: $N_{t}=1 \times 10^{21} \mathrm{~cm}^{-3}, \alpha^{-1}=1 \mathrm{~nm}, C_{i}=1.5 \times 10^{-4} \mathrm{~F} / \mathrm{m}^{2}, \sigma_{0} / \mathrm{k}_{\mathrm{B}} \mathrm{T}=2, E_{f 0}=-0.65$ $\mathrm{eV}, \varepsilon_{s}=3, \mathrm{P}=0 \mathrm{D}, V_{g^{-}} V_{f b}=5 \mathrm{~V}$, and $T=300 \mathrm{~K}$. The input parameters for Rubrene in inset are: $N_{t}=1.4 \times 10^{21} \mathrm{~cm}^{-3}, C_{i}=3 \times 10^{-5} \mathrm{~F} / \mathrm{m}^{2}, \sigma_{0} / \mathrm{k}_{\mathrm{B}} \mathrm{T}=1.3, E_{f 0}=-0.52 \mathrm{eV}$, and $T=294 \mathrm{~K}$, the other parameters are the same as those of PEDOT:Tos.

Figure 3. Gate-voltage dependence of the Seebeck coefficient for different dipole moments. The input parameters are: $N_{t}=1 \times 10^{20} \mathrm{~cm}^{-3}, \alpha^{-1}=0.5 \mathrm{~nm}, C_{i}=1 \times 10^{-4} \mathrm{~F} / \mathrm{m}^{2}$, $\sigma_{0} / \mathrm{k}_{\mathrm{B}} \mathrm{T}=4, E_{f 0}=-0.65 \mathrm{eV}, \varepsilon_{s}=3$, and $T=300 \mathrm{~K}$.

Figure 4. Seebeck coefficient as a function of dipole moment for different energetic disorder. The input parameters are $V_{g}-V_{f b}=3 \mathrm{~V}$ and $E_{f 0}=-0.52 \mathrm{eV}$, and the other parameters are the same as those in Fig. 3.

Figure 5. Energetic disorder dependent Seebeck coefficient for different dipole moment. The input parameters are $N_{t}=2 \times 10^{19} \mathrm{~cm}^{-3}, V_{g^{-}} V_{f b}=5 \mathrm{~V}, \alpha^{-1}=0.8 \mathrm{~nm}, C_{i}=1 \times 10^{-4}$ $\mathrm{F} / \mathrm{m}^{2}, \varepsilon_{s}=4, E_{f 0}=-0.52 \mathrm{eV}$ and $T=300 \mathrm{~K}$.

Figure 6. Temperature dependence of the Seebeck coefficient for different dipole moments and comparison between simulation and experimental results. The symbol and color solid lines are the experimental data and theoretical calculations, respectively. 
Figure 1

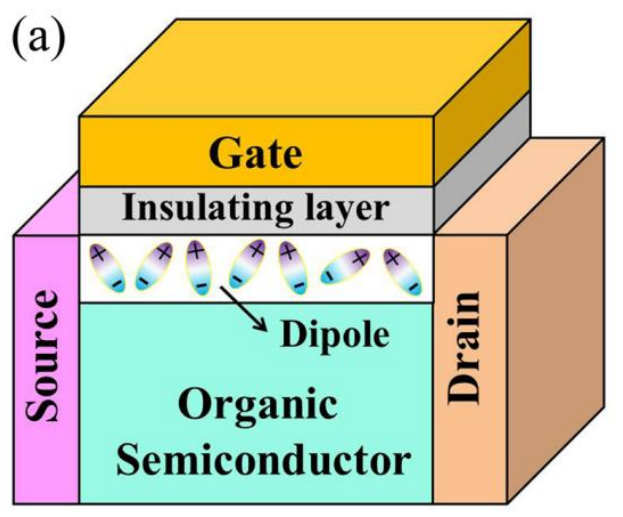

OTFT device structure

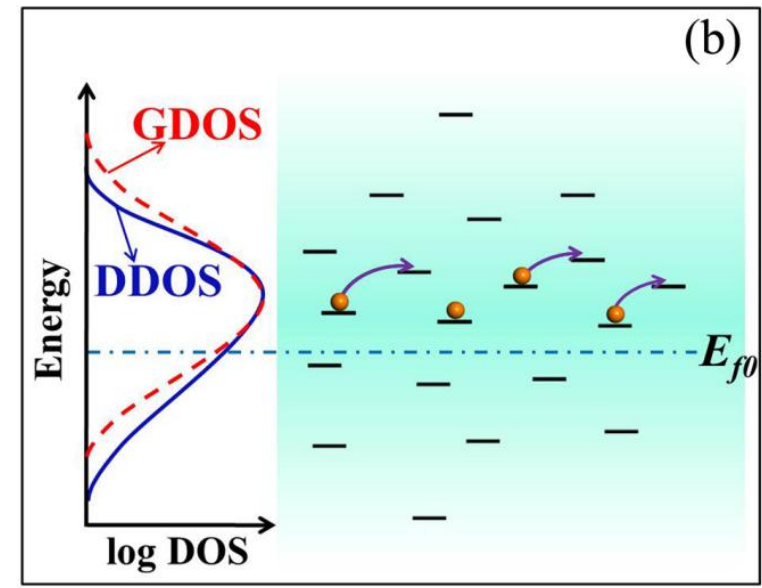


Figure 2

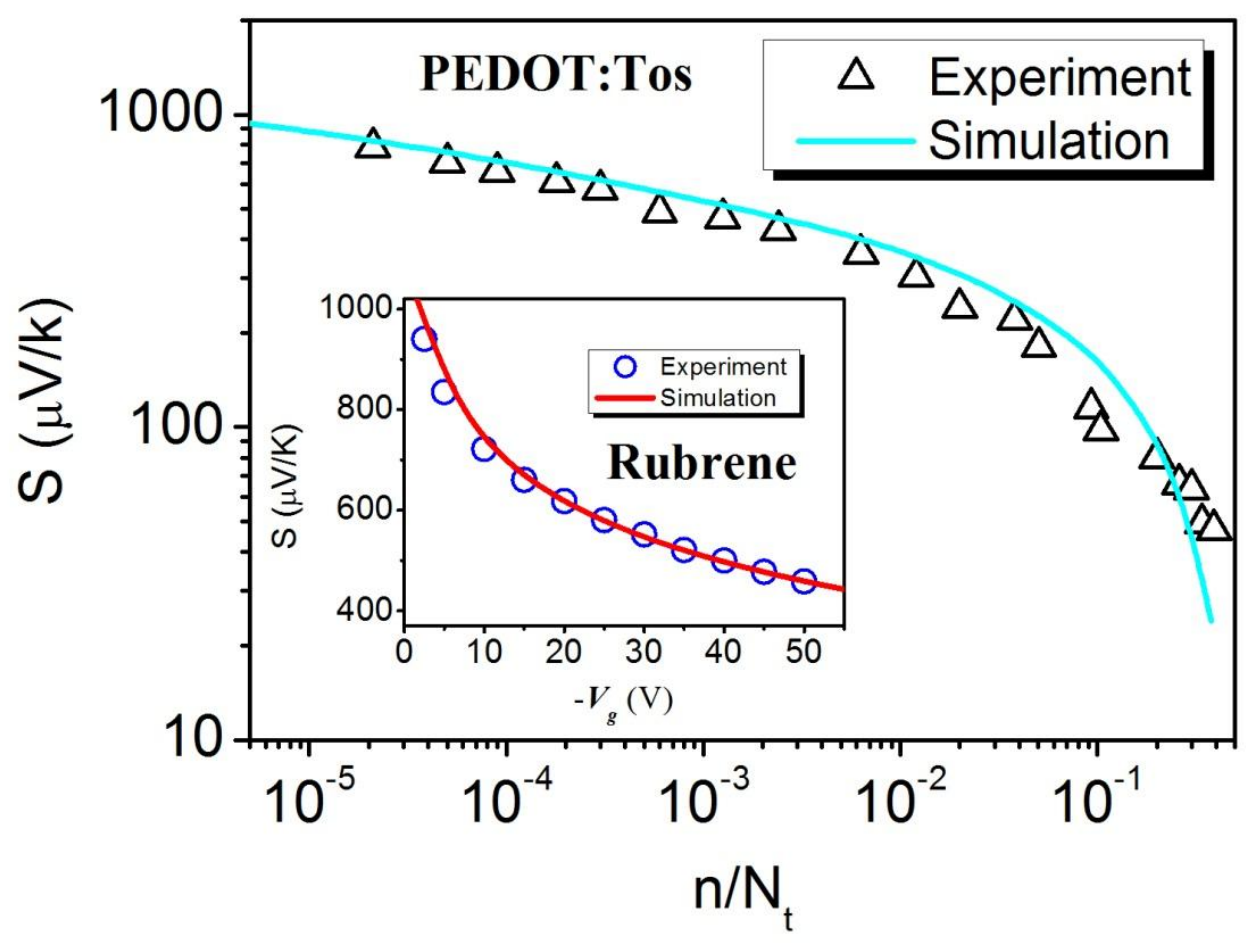


Figure 3

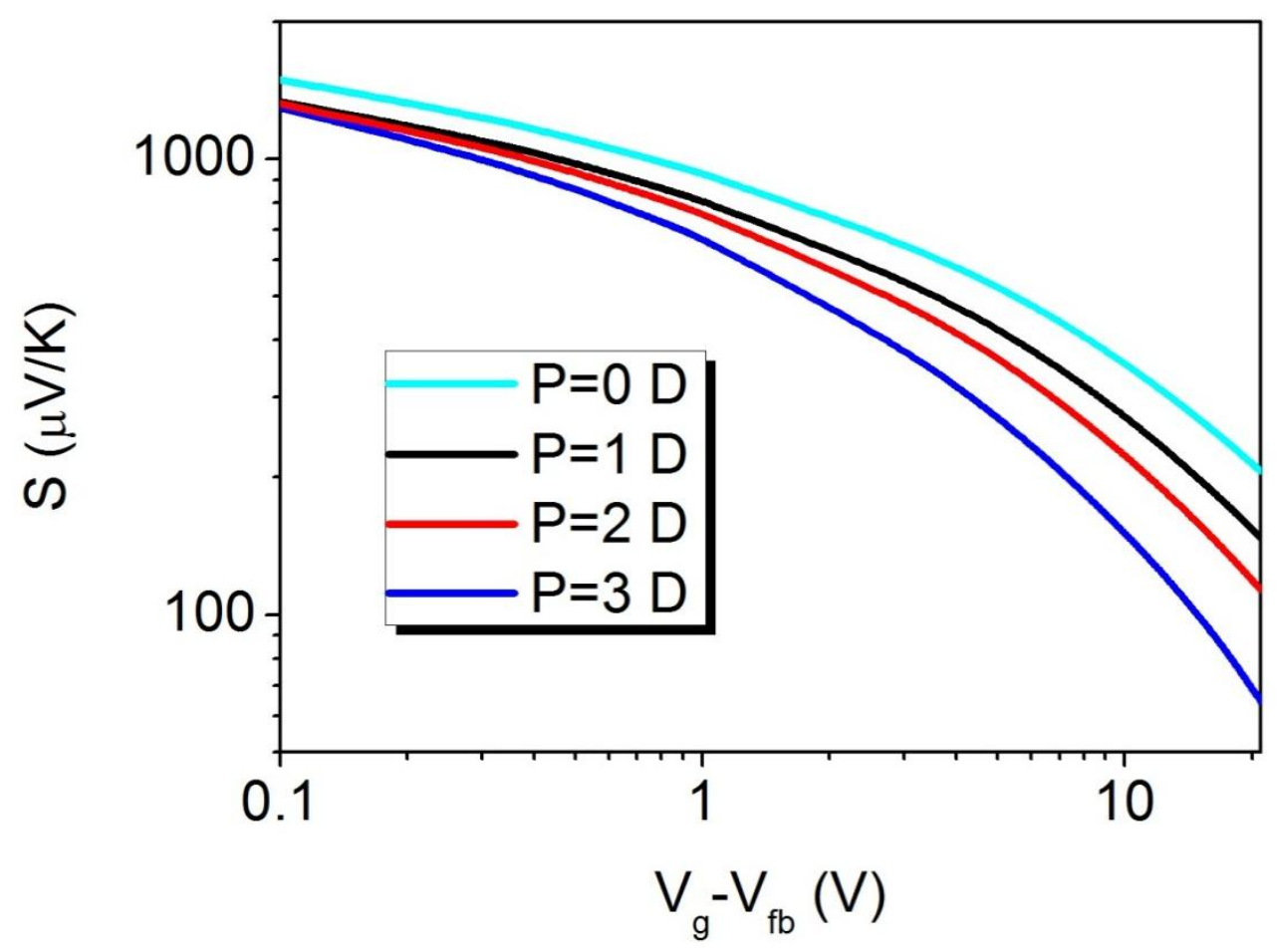


Figure 4

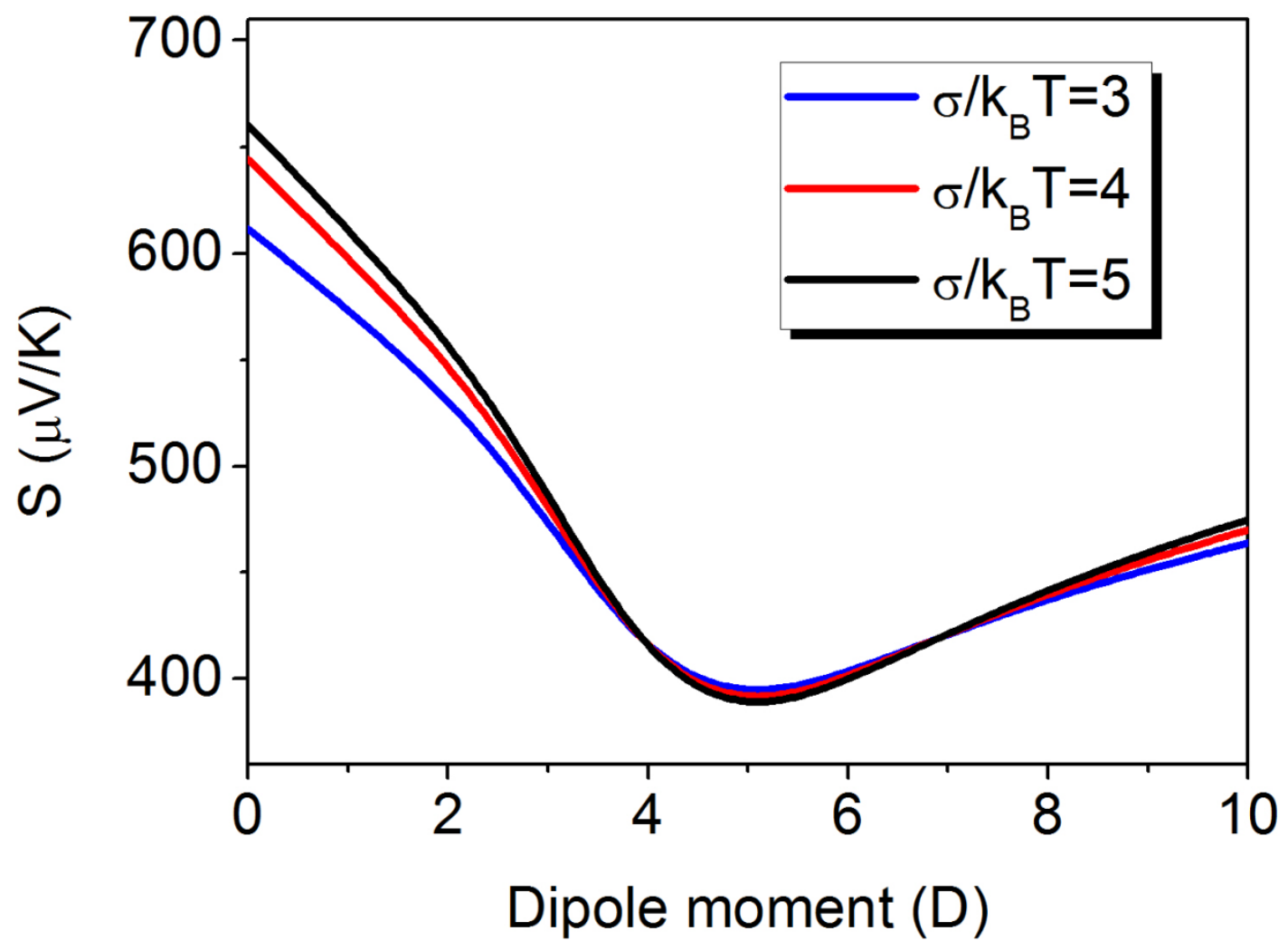


Figure 5

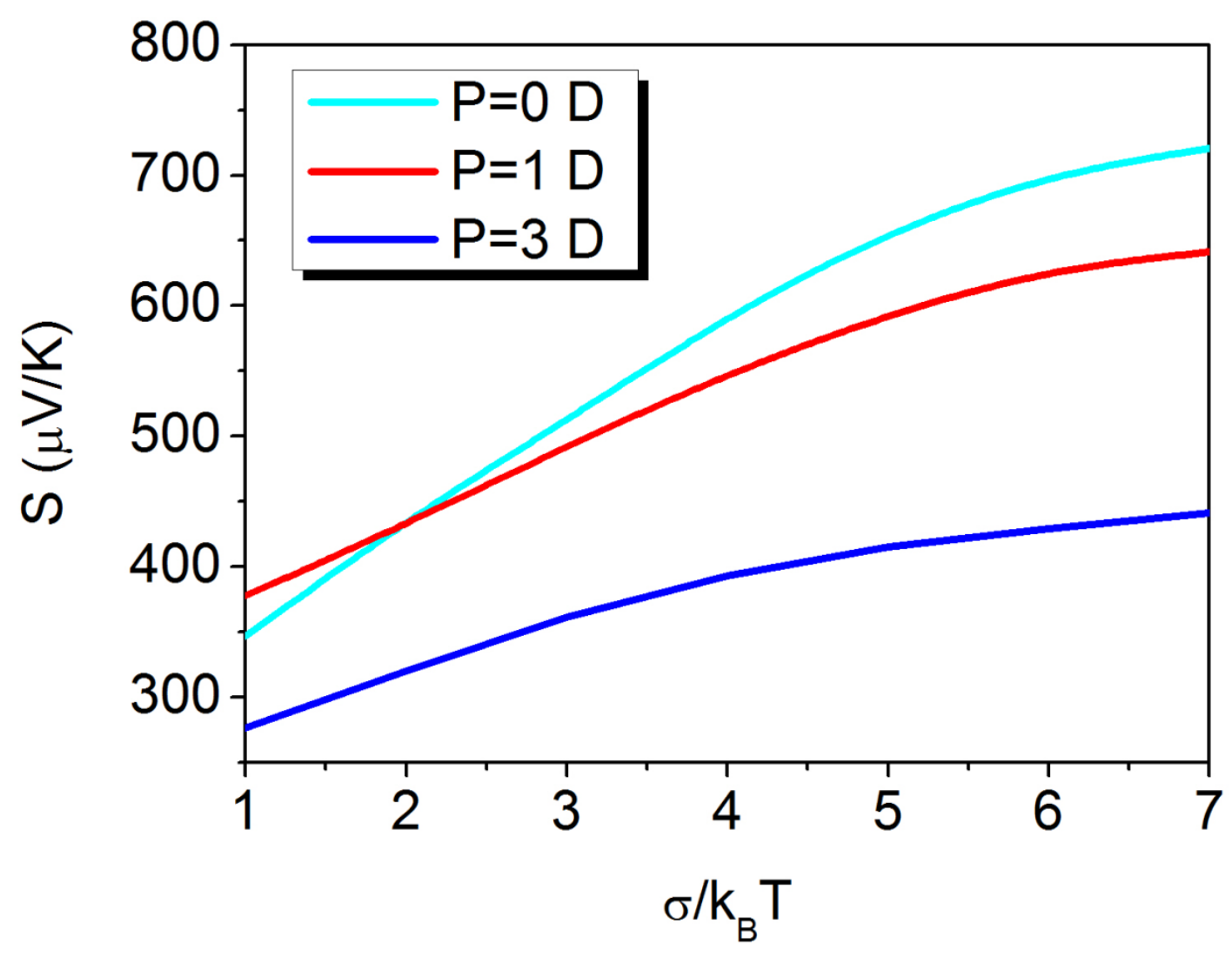


Figure 6

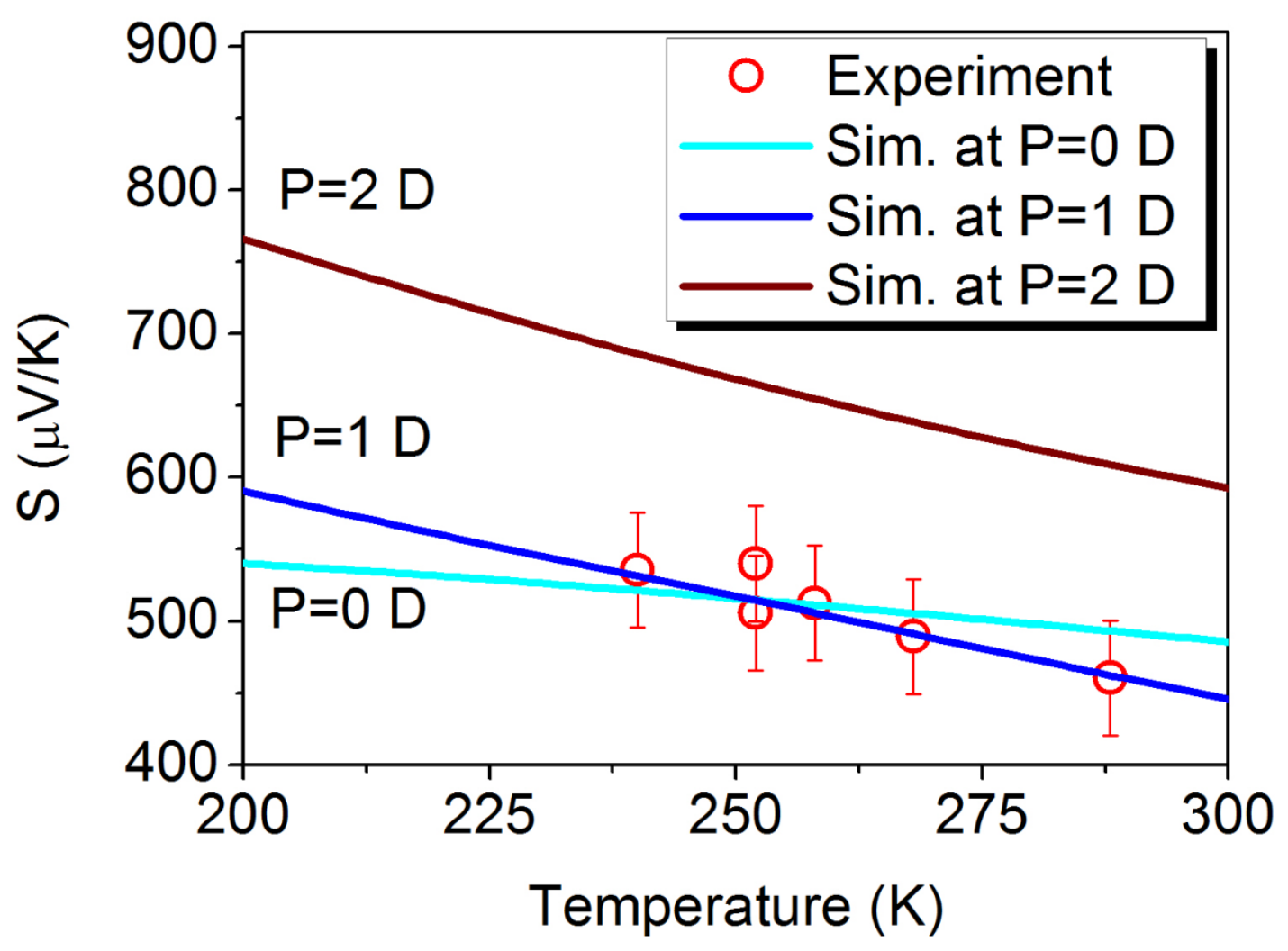




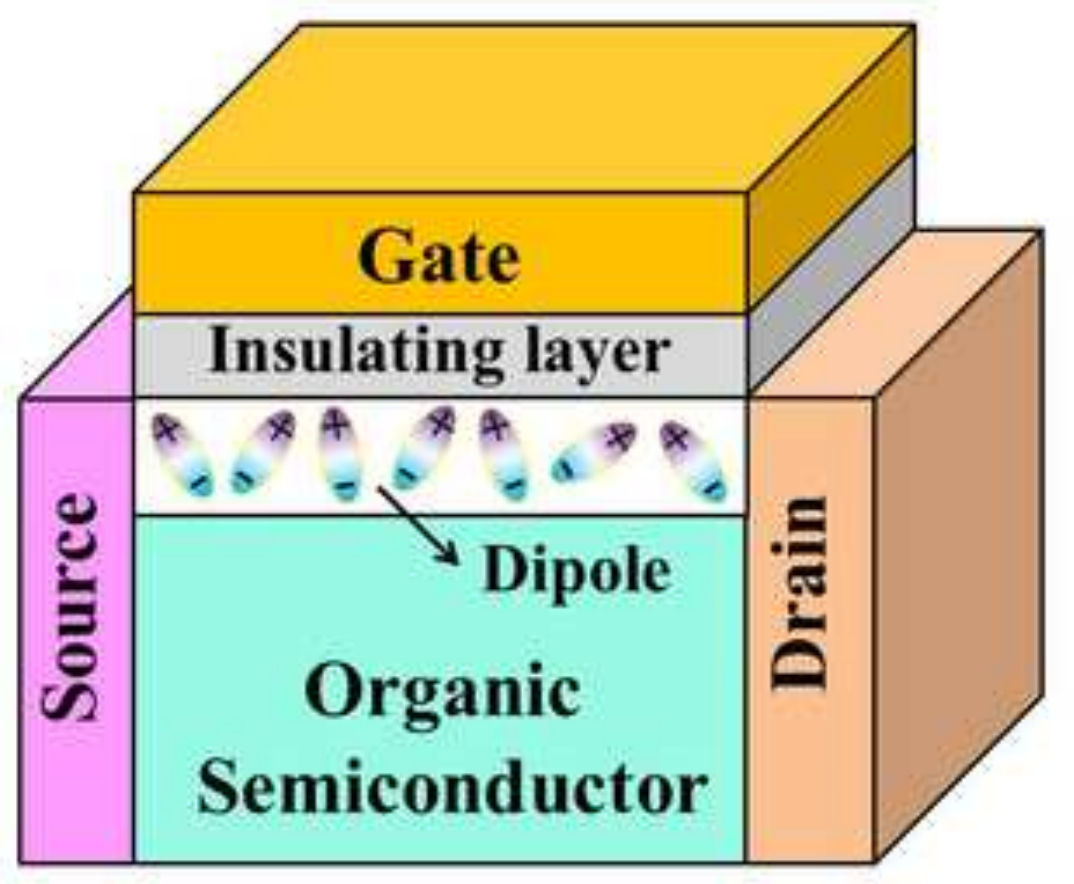

OTFT device structure

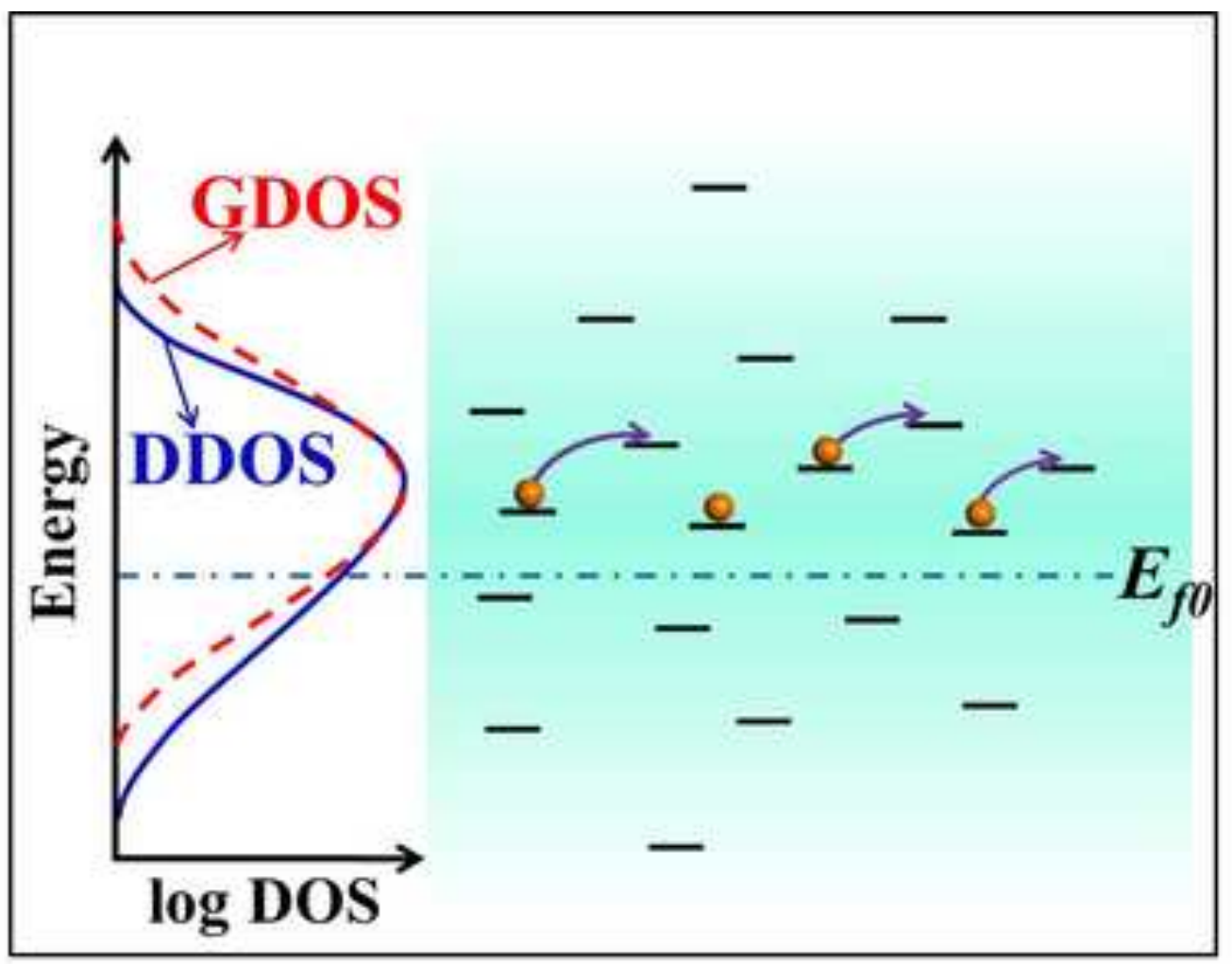

